Abstract 0P11 Table 1

\begin{tabular}{lllllllll}
\hline Normal ALT [ALT <30 IU/I for females and ALT <45 IU/I for males] \\
\hline Test & AUROC & Cut-off & Sens (\%) & Spec (\%) & PPV (\%) & NPV (\%) & Avoid biopsy \\
\hline AST/ALT & 0.81 & 0.8 & 94 & 44 & 35 & 96 & $18 / 70(26 \%)$ \\
BARD & 0.71 & 2 & 94 & 26 & 29 & 93 & $15 / 70(21 \%)$ \\
FIB-4 & 0.86 & 1.30 & 82 & 77 & 52 & 92 & $43 / 70(61 \%)$ \\
NAFLD & 0.85 & -1.455 & 82 & 51 & 35 & 90 & $29 / 70(41 \%)$ \\
Raised ALT & & & & & & & \\
AST/ALT & 0.79 & 0.8 & 59 & 86 & 46 & 91 & $184 / 235(78 \%)$ \\
BARD & 0.78 & 2 & 83 & 57 & 28 & 94 & $118 / 235(50 \%)$ \\
FIB-4 & 0.85 & 1.30 & 81 & 72 & 37 & 95 & $148 / 235(63 \%)$ \\
NAFLD & 0.80 & -1.455 & 71 & 65 & 29 & 92 & $138 / 235(59 \%)$ \\
\hline
\end{tabular}

Conclusion The FIB-4 score performed well in patients with normal or raised ALT, reliably excluding advanced fibrosis and reducing the need for liver biopsy. Therefore, the FIB-4 score may be an appropriate tool for use in primary care to triage patients with NAFLD who need referral for further assessment. Further validation in a general practice cohort is underway.

\section{Viral hepatitis}

\section{OP12 SIMILAR SVR RATES IN IL28B CC, CT OR TT PRIOR RELAPSER, PARTIAL- OR NULL-RESPONDER PATIENTS TREATED WITH TELAPREVIR/PEGINTERFERON/RIBAVIRIN: RETROSPECTIVE ANALYSIS OF THE REALIZE STUDY}

doi:10.1136/gutjnl-2011-300857b.12

${ }^{1} \mathrm{G}$ R Foster, ${ }^{2} \mathrm{~S}$ Pol, ${ }^{3} \mathrm{~J}$ Aerssens, ${ }^{4} \mathrm{~S}$ Zeuzem, ${ }^{5} \mathrm{P}$ Andreone, ${ }^{6} \mathrm{E} \mathrm{J}$ Lawitz, ${ }^{7} \mathrm{~S}$ Roberts, ${ }^{8} \mathrm{Z}$ Younossi, ${ }^{9} \mathrm{R}$ Focaccia, ${ }^{10} \mathrm{~A}$ Horban, ${ }^{11} \mathrm{P} \mathrm{J}$ Pockros, ${ }^{3} \mathrm{R}$ Van Heeswijk, ${ }^{3} \mathrm{~S}$ de Meyer, ${ }^{12} \mathrm{D}$ Luo, ${ }^{3} \mathrm{M}$ Beumont, ${ }^{12} \mathrm{G}$ Picchio. ${ }^{1}$ Institute of Cell and Molecular Science, Queen Mary University of London, London, UK; ${ }^{2}$ University Paris Descartes, INSERM UnitĖ 567, and Assistance Publique-Hopitaux de Paris, Cochin Hospital, Paris, France; ${ }^{3}$ Tibotec BVBA, Beerse, Belgium; ${ }^{4}$ Johann Wolfgang Goethe University Medical Center,

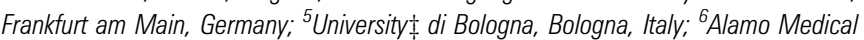
Research, Ltd, San Antonio, Texas, USA; 'Department of Gastroenterology, Alfred Hospital, Melbourne, Victoria, Australia; ${ }^{8}$ Center for Liver Disease, Inova Fairfax Hospital, Falls Church, Virginia, USA; ${ }^{9}$ Emilio Ribas Infectious Diseases Institute, $S_{\text {, }} 0$ Paulo, Brazil; ${ }^{10}$ Medical University of Warsaw, Wolska, Warsaw, Poland; ${ }^{11}$ Scripps Clinic and The Scripps Research Institute, La Jolla, California, USA; ${ }^{12}$ Tibotec Inc., Titusville, New Jersy, USA

Introduction IL28B polymorphisms are linked to differences in SVR rates in HCV treatment-naÔve patients treated with pegylated interferon (P) and ribavirin (R). REALIZE is a Phase 3 study that compared the efficacy, safety and tolerability of telaprevir $(\mathrm{T})$, with or without a Lead-in (LI), in combination with PR against PR alone in prior treatment-failure patients including relapsers, partial responders and null responders (NR). Both T/PR arms were superior to control in all three patient categories. The relationship between IL28B genotype and SVR was investigated retrospectively.

Method 527/662 (80\%) patients enrolled in REALIZE consented to genetic testing. This represented $72 \%, 76 \%$ and $98 \%$ of the total relapsers, partial responders, and NR, respectively. Genotype rs12979860 was determined using a TaqMan allelic discrimination assay validated against Sanger sequencing on 50 independent samples. This was a retrospective study based on patients who consented to genetic testing prior to the discovery of IL28B, thus, sample size was not based on formal statistical considerations.

Results Overall, 94\% were Caucasian and 4\% were Black. $18 \%$ of patients were IL28B CC, $61 \%$ CT and 21\% TT. By prior response category, the highest proportion of IL28B TT patients was among prior NR (28\%) while the highest frequency of CC patients occurred among prior relapsers (27\%). The observed IL28B genotype frequencies indicate that the population was not in Hardy-Weinberg equilibrium $\left(\chi^{2}=28, p<0.0001\right)$. IL28B genotypes were well balanced across all arms with exception of a higher frequency of TTs in the placebo arm. Since no differences were observed between the two T arms, a pooled analysis is presented.

Conclusion Differences in SVR rates among IL28B CC, CT and TT patients were only evident when the three patient subpopulations were pooled, however, SVR among CT and TT patients were still high. In this retrospective analysis, IL28B genotype did not contribute to outcome prediction in prior treatment-experienced patients treated with a telaprevir-based regimen and thus, may be of limited utility in this setting.

\section{OP13 CD8B LOW T CELLS ARE A PROMINENT, FUNCTIONALLY DISTINCT POPULATION IN CHRONIC HEPATITIS B INFECTION}

doi:10.1136/gutjnl-2011-300857b.13

${ }^{1} \mathrm{E}$ Marrinan, ${ }^{2} \mathrm{~L}$ Walker, ${ }^{3} \mathrm{E}$ Barnes, ${ }^{4} \mathrm{P}$ Klenerman. ${ }^{1}$ Peter Medawar Building for Pathogen Research, Nuffield Department of Medicine, University of Oxford; ${ }^{2}$ Peter Medawar Building for Pathogen Research; ${ }^{3}$ Nuffield Department of Medicine, ${ }^{4}$ University of Oxford

Introduction The failure of antigen-specific CD8 T cells is a well recognised feature of chronic hepatitis $\mathrm{B}(\mathrm{HBV})$ infection, however the bulk CD8 $\mathrm{T}$ cell population is also characterised by low expression of CD28 and poor IL-2 production and proliferation capacity (Das, Hoare et al 2008). We have previously observed a prominent $\mathrm{CD} 8 \mathrm{~T}$ cell population in chronic $\mathrm{HBV}$ to be $\mathrm{CD} 8 \mathrm{~b}^{\text {low }}$ (Walker, Kang et al) - this is most obvious in the cells which lack expression of CD161 (a molecule associated with liver homing). The relationship of these two observations has not previously been explored. Such changes may represent infection induced immune exhaustion or an acquired tolerance mechanism, which is breached during flares in disease activity. Both scenarios have important implications either for development of immunotherapy for viral clearance or treatment for prevention of disease progression.

Aim The aim of this study was to further characterise the phenotypic and functional features of the bulk CD8 T cell population in chronic HBV infection and the relationship of $\mathrm{CD} 288^{\text {low }} \mathrm{CD} 8+$ and CD161-CD8b ${ }^{\text {low }} \mathrm{T}$ cell populations.

Method Peripheral blood mononucleocytes were obtained from patients with eAg+ve chronic HBV ( $\mathrm{n}=6)$, eAg-ve chronic HBV $(n=12)$, chronic hepatitis C (HCV) genotype $1(n=5)$ and healthy

Abstract OP12 Table 1

\begin{tabular}{|c|c|c|c|c|c|c|c|c|}
\hline \multirow[b]{2}{*}{$\%$ SVR (n) } & \multicolumn{2}{|c|}{ Overall Population } & \multicolumn{2}{|l|}{ Prior Relapsers } & \multicolumn{2}{|c|}{ Prior Partial Responders } & \multicolumn{2}{|c|}{ Prior Null Responders } \\
\hline & $\begin{array}{l}\text { Pooled } \\
\text { T12/PR48 Arms } \\
\text { (N=422) }\end{array}$ & $\begin{array}{l}\text { Pbo/PR48 } \\
\text { Arm (N=105) }\end{array}$ & $\begin{array}{l}\text { Pooled } \\
\text { T12/PR48 Arms } \\
\text { (N=209) }\end{array}$ & $\begin{array}{l}\text { Pbo/PR48 } \\
\text { Arm }(\mathrm{N}=52)\end{array}$ & $\begin{array}{l}\text { Pooled } \\
\text { T12/PR48 Arms } \\
(\mathrm{N}=79)\end{array}$ & $\begin{array}{l}\text { Pbo/PR48 } \\
\text { Arm }(N=20)\end{array}$ & $\begin{array}{l}\text { Pooled } \\
\text { T12/PR48 Arms } \\
\text { (N=134) }\end{array}$ & $\begin{array}{l}\text { Pbo/PR48 } \\
\text { Arm (N=33) }\end{array}$ \\
\hline IL28B CC & $79(76)$ & $29(17)$ & $88(58)$ & $33(12)$ & $63(8)$ & $20(5)$ & $40(10)$ & $N A(0)$ \\
\hline IL28B CT & $60(266)$ & $16(58)$ & $86(117)$ & $20(30)$ & $58(57)$ & $20(10)$ & $29(92)$ & $6(18)$ \\
\hline IL28B TT & $61(80)$ & $13(30)$ & $85(34)$ & $30(10)$ & 71 (14) & $0(5)$ & 31 (32) & $7(15)$ \\
\hline
\end{tabular}


controls $(n=8)$. All patients were treatment naïve and had stable disease. FACS analysis was performed on both cell surface antibody staining using a panel of activation/exhaustion markers (CD25, CD38, CD69, HLA-DR, PD-1, CD8b) and intracellular cytokine staining following $\mathrm{PMA} /$ ionomycin stimulation. $\mathrm{CD} 8 \mathrm{~b}^{\text {high }} \mathrm{T}$ cells were freshly isolated from health controls and sorted using magnetic beads, prior to culture in a variety of conditions and subsequent FACS analysis.

Results In chronic HBV infection, regardless of eAg status, CD161$\mathrm{CD}_{8} \mathrm{~b}^{\text {low }} \mathrm{T}$ cells express significantly lower CD28 (eAg+ve $\mathrm{p}=0.005$, eAg-ve $p=0.0001$ ) and significantly higher HLA-DR (eAg+ve $p=0.002$, eAg-ve $p=0.009$ ) than their CD161-CD8b $b^{\text {high }}$ counterparts. The CD161-CD $8 b^{\text {low }} \mathrm{CD} 28^{\text {low }}{ }^{\mathrm{H}} \mathrm{A} A-\mathrm{DR} \mathrm{R}^{\text {high }}$ population represents a mean of $38 \%$ of total CD8 $\mathrm{T}$ cells in chronic HBV. The $\mathrm{CD} 161-\mathrm{CD} 8 \mathrm{~b}^{\text {low }} \mathrm{CD} 28^{\text {low }} \mathrm{HLA}-\mathrm{DR}{ }^{\text {high }}$ population produce significantly more IFN-g on stimulation with PMA/ionomycin than their CD8 $b^{\text {high }}$ CD28 ${ }^{\text {high }}$ HLA-DR ${ }^{\text {high }}$ counterparts $(p<0.0001)$. No difference was observed in IL-2 production. In addition we are able to demonstrate ready transition of sorted CD161-CD8b ${ }^{\text {high }}$ to CD161$\mathrm{CD}_{8} \mathrm{~b}^{\text {low }} \mathrm{T}$ cells from healthy controls cultured overnight in culture media alone, which provides an insight into the potential mechanisms involved in the generation of this population and an in-vitro model for use in further study.

Conclusion CD161-CD8b ${ }^{\text {low }}$ CD28 ${ }^{\text {low }} \mathrm{HLA}-\mathrm{DR}{ }^{\text {high }} \mathrm{T}$ cells are a functionally distinct, prominent $\mathrm{T}$ cell population in chronic HBV infection that are likely to profoundly influence the immune environment with important implications for the development of immunotherapy and treatment. The absence of this $\mathrm{T}$ cell subset in chronic HCV indicates a disease-specific rather than liver-related effect.

\section{REFERENCES}

1. Das A, Hoare $M$, et al. Functional skewing of the global CD8 $T$ cell population in chronic hepatitis B virus infection. J Exp Med 2008;205:2111-24.

2. Walker L, Kang Y. CD161 expressing CD8+ T-cells; elusive players in viral hepatitis. Gut 59(Suppl 2):A100.

\section{Basic science}

\section{OP14 IDENTIFICATION AND CHARACTERISATION OF A NOVEL LY- 6C ${ }^{\text {INTERMEDIATE }}$ INTRAHEPATIC MACROPHAGE POPULATION WHICH MEDIATES THE RESOLUTION OF LIVER FIBROSIS, IS INDUCED BY PHAGOCYTOSIS AND CAN BE MANIPULATED THERAPEUTICALLY IN VIVO}

doi:10.1136/gutjnl-2011-300857b.14

P Ramachandran, A Pellicoro, M A Vernon, L Boulter, R L Aucott, S J Forbes, J P Iredale. University of Edinburgh

Introduction Macrophages are critical for the progression and resolution of hepatic fibrosis. Studies have identified Ly-6C ${ }^{\text {hi }}$ macrophages as the pro-fibrogenic subset in mice. However, the identity of the pro-resolution hepatic macrophage population is unknown.

Aim We aimed to identify and characterise the macrophage population mediating the resolution of hepatic fibrosis.

Method We established a model of reversible murine hepatic fibrosis by administering 4 weeks of $\mathrm{CCl}_{4}$, followed by tissue harvests at serial timepoints after the final dose.

Results Histological analysis identified maximal fibrosis resolution between 72 and $96 \mathrm{~h}$ after the final $\mathrm{CCl}_{4}$ dose. Flow cytometry of hepatic macrophages showed that during maximal fibrosis resolution there was a loss of pro-fibrotic Ly- $6 \mathrm{C}^{\text {hi }}$ macrophages and large increase in a Ly- $6 C^{\text {intermediate }}$ macrophage population, which were the most numerous macrophage subset identified at any timepoint during fibrogenesis and recovery. Using CD11B-DTR mice, macrophages were depleted during the rapid resolution phase, resulting in a failure to remodel the hepatic scar. Critically, this depletion strategy selectively ablated the Ly-6C $\mathrm{C}^{\text {int }}$ subset, the degree of depletion correlating significantly with the amount of persistent fibrosis.
A series of bone marrow transplantation, adoptive transfer and in situ labelling experiments identified that the pro-resolution Ly-6 $\mathrm{C}^{\text {int }}$ macrophage population derives from recruitment of Ly- $6 \mathrm{C}^{\text {hi }}$ monocytes, a common origin to the pro-fibrotic Ly-6C ${ }^{\text {hi }}$ macrophages, indicative of a phenotypic switch in situ.

Microarray profiling of FACS sorted Ly- $6 C^{\text {int }}$ macrophages in comparison to pro-fibrotic Ly- $6 \mathrm{C}^{\text {hi }}$ macrophages, demonstrated a novel phenotype outside the M1/M2 macrophage paradigm, with down regulation of pro-inflammatory and pro-fibrotic genes, upregulation of matrix degrading enzymes and enrichment for phagocytosis related pathways. Confocal microscopy indicated that the Ly- $6 C^{\text {int }}$ population contained more intracellular apoptotic debris, confirming the post-phagocytic nature of these cells.

Feeding primary murine macrophages with hepatocyte debris in vitro induced a similar phenotypic switch to that seen in vivo. Furthermore, this phagocytosis-induced switch could be modelled by feeding macrophages with liposomes in vitro. Critically, systemic administration of liposomes to mice during maximal fibrosis resolution increased the number of hepatic Ly- $6 C^{\text {int }}$ macrophages and accelerated the resolution of fibrosis.

Conclusion In summary, we have identified the specific Ly- $6 \mathrm{C}^{\text {int }}$ macrophage subset which mediates the resolution of hepatic fibrosis. Extensive characterisation demonstrated this macrophage phenotype is produced by the phagocytosis of dead cells and thus can be manipulated in vivo by the induction of phagocytic behaviour with a beneficial effect on fibrosis resolution.

\section{OP15 DISCOVERY OF NEW LIVER FIBROSIS MARKERS IN HEPATITIS C PATIENTS USING PROTEOMICS}

doi:10.1136/gutjnl-2011-300857b.15

B Gangadharan, M Bapat, J Rossa, R Antrobus, D Chittenden, B Kampa, E Barnes, R A Dwek, N Zitzmann. Oxford Antiviral Drug Discovery Unit, Oxford Glycobiology Institute, Department of Biochemistry, University of Oxford, Oxford, UK

Introduction Liver biopsy is the reference standard for assessing liver fibrosis, and no reliable non-invasive diagnostic approach is available to discriminate between the intermediate stages of fibrosis. Therefore suitable serological biomarkers of liver fibrosis are urgently needed.

Aim The objective of this study was to use proteomics to identify novel fibrosis biomarkers in hepatitis $\mathrm{C}$ patients with different degrees of liver fibrosis.

Method Proteins in plasma samples from healthy control individuals and patients with hepatitis $\mathrm{C}$ virus (HCV) induced cirrhosis were analysed using a proteomics technique-two dimensional gel electrophoresis (2-DE). This technique separated the proteins in plasma samples of control and cirrhotic patients and by visualising the separated proteins we were able to identify proteins which were increasing or decreasing in hepatic cirrhosis. Identified markers were validated across all Ishak stages and compared to the markers used in FibroTest, Enhanced Liver Fibrosis (ELF) test, Hepascore and FIBROSpect by western blotting.

Results 44 candidate biomarkers for hepatic fibrosis were identified of which 20 were novel. western blot validation of all candidate markers using plasma samples from patients across all Ishak fibrosis scores showed that the markers which changed with increasing fibrosis most consistently included lipid transfer inhibitor protein, complement C3d, corticosteroid-binding globulin, apolipoprotein J and apolipoprotein L1. These five novel markers which are secreted in blood showed a promising consistent change with increasing fibrosis stage when compared to the markers used for the FibroTest, ELF test, Hepascore and FIBROSpect.

Conclusion This study identifies 20 novel fibrosis biomarker candidates. The proteins identified by these improved approaches may help to assess hepatic fibrosis and eliminate the need for invasive liver biopsies. 